\title{
The minimum mass for opacity-limited fragmentation in turbulent cloud cores
}

\begin{abstract}
D. F. A. Boyd and A. P. Whitworth
Department of Physics and Astronomy, Cardiff University, PO Box 913, 5 The Parade, Cardiff CF24 3YB, Wales, UK e-mail: [D.Boyd;A.Whitworth]@astro.cf.ac.uk

Received 21 July 2004 / Accepted 4 October 2004

Abstract. We present a new analysis of the minimum mass for star formation, based on opacity-limited fragmentation. Our analysis differs from the standard one, which considers hierarchical fragmentation of a 3D medium, and yields $M_{\text {MIN }} \sim$ 0.007 to $0.010 M_{\odot}$ for Population I star formation. Instead we analyse the more realistic situation in which there is one-shot fragmentation of a shock-compressed layer, of the sort which arises in turbulent star-forming clouds. In this situation, $M_{\mathrm{MIN}}$ can be smaller than $0.003 M_{\odot}$. Our analysis is more stringent than the standard one in that (a) it requires fragments to have condensation timescales shorter than all competing mass scales, and (b) it takes into acount that a fragment grows by accretion whilst it is condensing out, and therefore has to radiate away the energy dissipated in the associated accretion shock (in addition to the $P \mathrm{~d} V$ work done by internal compression). It also accords with the recent detection, in young star clusters, of free-floating star-like objects having masses as low as $0.003 M_{\odot}$.
\end{abstract}

Key words. stars: formation - ISM: clouds - turbulence -hydrodynamics

\section{Introduction}

3-dimensional opacity-limited hierarchical fragmentation yields a minimum stellar mass $M_{\text {MIN3 }} \sim 0.007$ to $0.010 M_{\odot}$. This result was first obtained to order of magnitude by Rees (1976), but the range quoted above is the result of more detailed treatments of the thermodynamics of fragmentation by Low \& Lynden-Bell (1976), Silk (1977) and Boss (1988). However, there are problems with the process of 3-dimensional hierarchical fragmentation, and this estimate of $M_{\mathrm{MIN} 3}$ almost certainly needs to be revised upwards (see Sect. 2). Moreover, it is now generally accepted (e.g. Elmegreen 2000; Pringle et al. 2001; Hartmann et al. 2001; Elmegreen 2002; Padoan \& Nordlund 2002; Vázquez-Semadeni et al. 2003) that starforming clouds are transient turbulent entities, and that star formation occurs almost as soon as a cloud forms, within a few crossing times. In this picture, prestellar cores are created where turbulent elements collide with sufficient ram-pressure for the resulting shock-compressed layer to be gravitationally unstable. The process of fragmentation is then quite different from that envisaged in 3-dimensional hierarchical fragmentation. In particular, (i) the fragmentation of a shock-compressed layer can, and usually does, proceed whilst the layer is still accumulating (and therefore it is confined by ram pressure); (ii) the convergent motions leading to the formation of a fragment are initially concentrated in the plane of the shocked layer, i.e. fragmentation of a shock-compressed layer is essentially two-dimensional.

In this paper, we present a model to describe the 2-dimensional opacity-limited fragmentation of a shockcompressed layer and use this model to determine the minimum mass of the fragments that can condense out of such a layer, $M_{\mathrm{MN} 2}$. Section 2 reviews the standard paradigm of 3-dimensional hierarchical fragmentation, in which a cloud fragments into smaller and smaller subclouds until radiative cooling can no longer match the compressional heating rate and the the subclouds become approximately adiabatic. In Sect. 3 we highlight the differences between hierarchical 3-dimensional fragmentation and the fragmentation of a 2-dimensional layer. We derive equations to describe how a shock-compressed layer is formed by the collision of two streams of gas, and how the layer fragments whilst it is still forming. In Sect. 4 we use this model to follow the evolution of a fragment and hence to estimate the minimum mass for a fragment. In Sect. 5 we briefly summarize our results.

Observational and numerical evidence for the formation and fragmentation of shock-compressed layers, in regions of imminent or ongoing star formation, is hard to identify. This contrasts with the substantial evidence for the formation and fragmentation of filaments in such regions. Nonetheless, we believe that the formation and fragmentation of shockcompressed layers must be the fundamental process in turbulent star formation regions. First (from a theoretical perspective), to create a filament ab initio would require a very contrived flow geometry. Second (again from a theoretical perspective), the fragmentation of a layer normally proceeds via the formation of a network of filaments (e.g. Turner et al. 1995; Whitworth et al. 1995; Bhattal et al. 1998); this is because of the well established theorem (e.g. Zeldovich 1979) which asserts that if gravitational contraction gets ahead in one dimension (due either to a greater initial perturbation, or less 
resistance), it tends to get further ahead. Third (from an observational perspective), layers are much harder to identify than filaments, unless they are seen edge-on, in which case they look like filaments.

Moreover, free-floating objects with masses as low as $0.003 M_{\odot}$ have now been detected in young star clusters, like $\sigma$ Orionis (Zapatero Osorio et al. 2002), and it beholds us to ask how they might have formed. They cannot form by three-dimensional fragmentation, but it appears that they could form by layer fragmentation, as analyzed here. Other possible formation mechanisms include the photo-erosion of preexisting massive cores (Hester 1997; Whitworth \& Zinnecker 2004) and impulsive interactions between discs in dense clusters (Boffin et al. 1998; Watkins et al. 1998a,b).

\section{Three-dimensional hierarchical fragmentation}

Theories of star formation have traditionally been based on the idea of opacity-limited hierarchical fragmentation (Hoyle 1953). A massive, 3-dimensional, Jeans-unstable cloud starts to contract and, as long as the isothermal sound speed, $a$, remains constant, the Jeans mass

$M_{\mathrm{JEANS} 3} \simeq\left[\frac{375 a^{6}}{4 \pi G^{3} \rho}\right]^{1 / 2} \sim \frac{6 a^{3}}{G^{3 / 2} \rho^{1 / 2}}$,

decreases with increasing density, $\rho$. Thus, once the original cloud has contracted sufficiently, the Jeans mass $M_{\text {JEANS3 }}$ is reduced and the cloud can fragment into subclouds. Once these subclouds have contracted sufficiently, they themselves can fragment into still smaller "sub-subclouds", and so on. The process can continue recursively, breaking the original cloud up into ever smaller fragments, as long as the isothermal sound speed remains approximately constant, i.e. as long as the contracting gas is able to radiate away, immediately, the $P \mathrm{~d} V$ work being done on it by compression. Once the smallest fragments become so dense and opaque to their own cooling radiation that they can no longer radiate away the $P \mathrm{~d} V$ work immediately, the sound speed starts to increase, the Jeans mass decreases no further, and fragmentation therefore stops.

The minimum mass for star formation can be found from a general analysis of this process, as shown by Rees (1976); for simplicity we neglect all purely numerical factors. A fragment of radius $R$, which has just become Jeans unstable and started to condense out, contracts at speed $\mathrm{d} R / \mathrm{d} t \sim-a$. Hence the heating rate due to $P \mathrm{~d} V$ work is

$\mathcal{H}=-P \frac{\mathrm{d} V}{\mathrm{~d} t} \sim-\rho a^{2} R^{2} \frac{\mathrm{d} R}{\mathrm{~d} t} \sim \rho a^{3} R^{2}$,

where we have substituted $P=\rho a^{2}$ and $\mathrm{d} V / \mathrm{d} t \sim R^{2} \mathrm{~d} R / \mathrm{d} t$. If the fragment is to remain approximately isothermal, this must be less than the maximum possible radiative cooling rate,

$C \sim R^{2} \sigma_{\mathrm{SB}} T^{4}$

where $\sigma_{\mathrm{SB}}$ is the Stefan Boltzman constant and $T$ is the temperature. This maximum cooling rate will only be realized if the fragment cools like a blackbody, i.e. if it is optically thick but only just; detailed calculations (e.g. Low \& Lynden-Bell 1976) indicate that this is usually the case. To ensure that $C>\mathcal{H}$, i.e. that cooling is sufficently rapid to keep $a$ constant, we require

$\rho \lesssim \rho_{\mathrm{MAX}} \sim \frac{\sigma_{\mathrm{SB}} T^{4}}{a^{3}}$

and hence

$M \gtrsim M_{\mathrm{MIN} 3} \sim \frac{a^{3}}{G^{3 / 2} \rho_{\mathrm{MAX}}^{1 / 2}} \sim c\left[\frac{h}{G}\right]^{3 / 2}\left[\frac{k_{\mathrm{B}} T}{\bar{m}^{9}}\right]^{\frac{1}{4}}$,

or equivalently

$M \gtrsim M_{\mathrm{MIN} 3} \sim \frac{m_{\mathrm{PLANCK}}^{3}}{\bar{m}^{2}}\left[\frac{a}{c}\right]^{1 / 2}$.

Here we have substituted $a=\left(k_{\mathrm{B}} T / \bar{m}\right)^{1 / 2}$, where $k_{\mathrm{B}}$ is Boltzmann's constant and $\bar{m}$ is the mean gas-particle mass; $\sigma_{\mathrm{SB}} \sim k_{\mathrm{B}}^{4} / c^{2} h^{3}$, where $c$ is the speed of light and $h$ is Planck's constant; and $m_{\text {PLANCK }}=[\mathrm{ch} / G]^{1 / 2}$ is the Planck mass ${ }^{1}$.

However, there are problems with the notion of hierarchical fragmentation. Observationally, there is no clear evidence for it occuring, and from a theoretical viewpoint, it does not seem to work because the time-scale on which a subcloud condenses out is always longer than the time-scale on which its parent cloud is contracting.

Specifically, the condensation timescale in $3 \mathrm{D}$ is given by

$t_{\mathrm{COND} 3} \simeq t_{\mathrm{FF} 3}\left\{1-\left[\frac{M_{\mathrm{JEANS} 3}}{M}\right]^{2 / 3}\right\}^{-1 / 2}$,

where $t_{\mathrm{FF} 3}=(3 \pi / 32 G \rho)^{1 / 2}$ is the freefall time in 3D. Thus a parent cloud is always closer to freefall collapse than its subclouds. If we assume that the whole of the parent cloud breaks up into subclouds, so that at their inception the subclouds are touching, then a subcloud has insufficient time to establish itself as a distinct entity before it gets merged with neighbouring subclouds by the overall contraction of the parent cloud. For example, a subcloud with $M \simeq 4 M_{\text {JEANS3 }}$ condenses out on a timescale $\sim 1.3 t_{\mathrm{FF} 3}$ (and smaller subclouds condense out even more slowly), whereas the parent cloud contracts on a timescale $\sim t_{\mathrm{FF} 3}$.

If, instead, we assume that at their inception the subclouds are not touching, we must consider whether a subcloud continues to grow by accreting material from its surroundings as it condenses out. If we take at face value the Bondi formula for the rate of spherically symmetric accretion onto a mass $M$, from a background medium having density $\rho$ and isothermal sound speed $a$,

$\frac{\mathrm{d} M}{\mathrm{~d} t}=\frac{\mathrm{e}^{3 / 2} \pi G^{2} \rho M^{2}}{a^{3}}$

(Bondi 1952), we can rewrite this equation in the form

$\frac{\mathrm{d} \ell n(M)}{\mathrm{d}\left[t / t_{\mathrm{FF} 3}\right]} \simeq 42\left[\frac{M}{M_{\mathrm{JEANS} 3}}\right]$.

${ }^{1}$ Equation (6) shows that $M_{\mathrm{MIN} 3}$ is much smaller than the Chandrasekhar mass by virtue of (i) the mean gas-particle mass $\bar{m}$ being much larger than the mean mass per electron $\left(2 m_{\mathrm{PROTON}} /(1+X)\right.$, where $X$ is the fractional abundance of hydrogen by mass); and (ii) the sound speed, $a$, being much less than the speed of light, $c$. 
Thus, even though the Bondi scenario is not strictly applicable, the implication is that the subcloud mass will increase by a very large factor whilst it is condensing out (e.g. a fragment with initial mass $4 M_{\text {JEANS3 }}$ notionally increases its mass by a factor of $42 \times 4 \times 1.3 \simeq 220$ ). We must conclude that the initial fragment mass will be a significant underestimate of the final fragment mass.

\section{Fragmentation of a two-dimensional layer}

There is an alternative paradigm to hierarchical fragmentation, which overcomes these problems and agrees better with observation. In this paradigm (e.g. Larson 1981; Elmegreen 2000; Pringle et al. 2001; Hartmann et al. 2001; Padoan \& Nordlund 2002; Elmegreen 2002; Vázquez-Semadeni et al. 2003; Mac Low \& Klessen 2004), giant molecular clouds are relatively short-lived objects which form and dissolve on a dynamical timescale and have a turbulent and inhomogeneous internal structure. The substructure within a molecular cloud is therefore highly transient, with clumps forming, dispersing, and re-forming on a dynamical timescale, without necessarily spawning new stars. Only occasionally will a particularly dense, massive and strongly converging shock lead to the formation of a prestellar core. In this scenario, both core formation and core collapse are triggered by the collision of two turbulent elements of gas and the formation of a gravitationally unstable, shock-compressed layer. This is a one-step, two-dimensional fragmentation process: "one step" because the process does not repeat itself hierarchically; "two-dimensional" because, when the layer fragments, the wavelengths of the most rapidly growing fragments are much larger than the thickness of the layer (Whitworth et al. 1994a,b).

\subsection{Linear fragmentation of a static layer in plane-parallel symmetry}

2-dimensional fragmentation of a static layer (e.g. Larson 1985 ) is fundamentally different from 3-dimensional fragmentation. The Jeans mass is given by

$M_{\mathrm{JEANS} 2} \simeq \frac{9 a^{4}}{16 \pi G^{2} \Sigma} \sim \frac{0.2 a^{4}}{G^{2} \Sigma}$

where $\Sigma$ is the surface density of the layer; and the timescale on which Jeans unstable fragments condense out of the layer is given by

$t_{\mathrm{COND} 2} \simeq \frac{a}{\pi G \Sigma}\left\{\left[\frac{M_{\mathrm{JEANS} 2}}{M}\right]^{1 / 2}-\left[\frac{M_{\mathrm{JEANS} 2}}{M}\right]\right\}^{-1 / 2}$.

Since $t_{\mathrm{COND} 2}$ has a minimum for $M \simeq 4 M_{\mathrm{JEANS} 2}$, fragments on this mass scale condense out faster than smaller fragments and faster than larger fragments. Therefore $4 M_{\text {JEANS2 }}$ is a preferred mass scale for fragmentation. Fragmentation on this scale is likely to be permanent, because the fragments will not be merged by the overall contraction of the parent layer on larger scales. Given these differences between hierarchical fragmentation of a 3-dimensional cloud and one-step fragmentation of a 2-dimensional layer, we are interested in

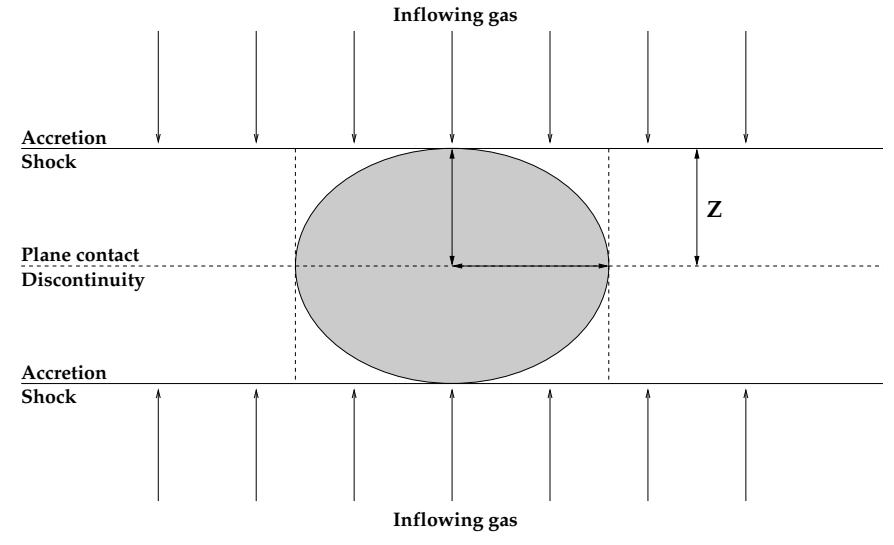

Fig. 1. Gas streams having density $\rho$ and sound speed $a$ collide to create a shock-compressed layer. As gas continues to flow into the layer from each side at speed $\pm v$, a spheroidal fragment of initial radius $r_{\text {INIT }}$ and initial height $z_{\mathrm{INIT}}=Z$ begins to condense out.

exploring how, and at what mass scale, opacity limits the fragmentation of a 2-dimensional layer. Is the minimum mass formed at the opacity limit significantly different from that estimated for 3-dimensional fragmentation?

\subsection{A shock-compressed layer in plane-parallel symmetry}

We consider two identical streams of gas with uniform density $\rho$ and uniform isothermal sound speed $a$, which collide head-on at relative speed $2 v$ to form a layer. We assume that a plane contact-discontinuity forms where the two streams meet and we fix our main coordinate frame in this contact discontinuity. The two streams approach the contact discontinuity with velocities $\pm v$, and a plane-parallel layer forms symmetrically about the discontinuity, bounded by two accretion shocks, as illustrated in Fig. 1. For simplicity we assume that radiative cooling in the shocked gas is so efficient that the gas rapidly cools back to its pre-shock temperature. If the full thickness of the layer is $2 Z(t)$, then the gas flows into the accretion shock at speed $u_{\mathrm{IN}}=v+\dot{Z}$, where $\dot{Z}=\mathrm{d} Z / \mathrm{d} t$, and out at speed $u_{\text {out }}=\dot{Z}$; note that $u_{\mathrm{IN}}$ and $u_{\mathrm{OUT}}$ are measured relative to the shock-front. Applying the isothermal shock condition,

$\frac{u_{\mathrm{IN}}}{a}=\frac{a}{u_{\mathrm{OUT}}}$,

we obtain

$\frac{v+\dot{Z}}{a}=\frac{a}{\dot{Z}}$,

whence

$\dot{Z}=\frac{\left[v^{2}+4 a^{2}\right]^{1 / 2}-v}{2}$.

The post-shock density (i.e. the density in the shock compressed layer) is

$\rho_{\text {SHOCKED }}=\rho \frac{u_{\mathrm{IN}}}{u_{\mathrm{OUT}}}=\rho \frac{\left[v^{2}+4 a^{2}\right]^{1 / 2}+v}{\left[v^{2}+4 a^{2}\right]^{1 / 2}-v}$, 
and so the surface density $\Sigma$ of the layer grows linearly with time according to

$\Sigma(t)=2 \rho_{\text {SHOСKED }} \dot{Z} t=\rho\left\{\left[v^{2}+4 a^{2}\right]^{1 / 2}+v\right\} t$.

As long as $v \gg a$, we can approximate

$\dot{Z} \simeq \frac{a^{2}}{v} \ll a$

$\rho_{\mathrm{SHOCKED}} \simeq \frac{\rho v^{2}}{a^{2}}$

$\Sigma(t) \simeq 2 \rho v t$

and so the sound-crossing time of the layer, $Z / a$, is much smaller than its growth time, $Z / \dot{Z}$. This means that the layer has sufficient time to relax and remain close to hydrostatic equilibrium. We also note that, provided

$t<\frac{1}{2(G \rho)^{1 / 2}}$

$G \Sigma^{2} \ll \rho_{\text {SHосKED }} a^{2}$, and thus self-gravity plays a negligible role compared with ram pressure in confining the layer in the direction perpendicular to the contact discontinuity. Consequently, the plane-parallel hydrostatic equilibrium of the layer has a very flat density profile, with little density contrast between the centre and the edge.

\subsection{Linear fragmentation of the layer}

Now we consider a small circular patch of radius $r$ on the layer, and consider whether it is able to condense out. The radial motion of the patch is determined by a competition between the hydrostatic acceleration $\sim a^{2} / r$ (which promotes expansion), and the self-gravitational acceleration $\sim \pi G \Sigma$ (which promotes contraction). Therefore we can write

$\ddot{r} \simeq \frac{a^{2}}{r}-\pi G \Sigma$,

and contraction $(\ddot{r}<0)$ requires

$r>R_{\mathrm{JEANS} 2} \simeq \frac{a^{2}}{\pi G \Sigma}$

The time-scale for condensation of a Jeans-unstable fragment is given by

$t_{\mathrm{COND2}} \simeq\left[\frac{r}{-\ddot{r}}\right]^{1 / 2} \simeq \frac{a}{\pi G \Sigma}\left\{\left[\frac{R_{\mathrm{JEANS} 2}}{r}\right]-\left[\frac{R_{\mathrm{JEANS} 2}}{r}\right]^{2}\right\}^{-1 / 2}$,

so the fastest condensing fragment has initial radius and condensation time-scale

$r_{\mathrm{FASTEST}} \simeq 2 R_{\mathrm{JEANS} 2} \simeq \frac{2 a^{2}}{\pi G \Sigma}$,

$t_{\text {FASTEST }} \simeq \frac{2 a}{\pi G \Sigma}$

As the layer piles up, $\Sigma$ increases, and so the size of the fastest condensing fragment decreases, and its condensation time-scale decreases. Condensation into the non-linear regime starts only when $t_{\text {FASTEST }} \lessgtr t$, and substituting for $\Sigma$ in Eq. (25), from Eq. (16), this condition gives the fragmentation time,

$t_{\mathrm{FRAG}}=\left\{\frac{2 a}{\pi G \rho\left\{\left[v^{2}+4 a^{2}\right]^{1 / 2}+v\right\}}\right\}^{1 / 2}$.

The mean radius and mass of the fragments which condense out are therefore

$r_{\mathrm{FRAG}} \equiv r_{\mathrm{FASTEST}}\left(t_{\mathrm{FRAG}}\right)=\left\{\frac{2 a^{3}}{\pi G \rho\left[\left(v^{2}+4 a^{2}\right)^{1 / 2}+v\right]}\right\}^{1 / 2}$,

$m_{\mathrm{FRAG}}=\pi r_{\mathrm{FRAG}}^{2} \Sigma\left(t_{\mathrm{FRAG}}\right)=\left\{\frac{2^{3} a^{7}}{\pi G^{3} \rho\left[\left(v^{2}+4^{2}\right)^{1 / 2}+v\right]}\right\}^{1 / 2}$

We note that

$\frac{2 r_{\mathrm{FRAG}}}{2 Z\left(t_{\mathrm{FRAG}}\right)} \simeq \frac{2 a}{\left[\left(v^{2}+4 a^{2}\right)^{1 / 2}-v\right]} \sim \frac{v}{a}$,

where the final expression obtains in the limit $v \gg a$. Therefore, at its inception, the mean diameter of a fragment is much larger than the thickness of the shock-compressed layer.

\subsection{Non-linear fragmentation of the layer}

The linear analysis above does not take into account the material which continues to flow into the shock layer, after it has started to fragment. It also does not describe how the fragment develops as it condenses out.

In order to follow the condensation of fragments into the non-linear regime, we model them as uniform density spheroids with radius $r$ and half-height $z$. The excursions of an isolated oblate spheroid of mass $m$, subjected to external pressure $P_{\text {EXT }}$, are governed by the equations

$$
\begin{aligned}
\ddot{r} \simeq-\frac{3 G m}{2}\left\{\frac{r \cos ^{-1}(z / r)}{\left(r^{2}-z^{2}\right)^{3 / 2}}-\frac{(z / r)}{\left(r^{2}-z^{2}\right)}\right\} & \\
& -\frac{20 \pi P_{\mathrm{EXT}} r z}{3 m}+\frac{5 a^{2}}{r},
\end{aligned}
$$

$$
\begin{aligned}
\ddot{z} \simeq-3 G m\left\{\frac{1}{\left(r^{2}-z^{2}\right)}-\frac{z \cos ^{-1}(z / r)}{\left(r^{2}-z^{2}\right)^{3 / 2}}\right\} \\
\quad-\frac{20 \pi P_{\mathrm{EXT}} r^{2}}{3 m}+\frac{5 a^{2}}{z} .
\end{aligned}
$$

In Eqs. (30) and (31), the first term on the righthand side represents self-gravity, the second term represents external pressure, and the third term represents internal pressure. Similar expressions describe the excursions of a prolate spheroid.

For an oblate spheroidal fragment condensing out of a shock-compressed layer, while the layer continues to accrete matter from the converging flows which are forming it, we must modify these equations to take into account two facts. (i) The external pressure is the ram pressure of the inflowing gas; (ii) the spheroid grows in mass as a consequence of the inflowing gas. Knowing $\dot{z}$, which is the speed with which the gas already 
in the spheroid expands, we can calculate how fast, $\dot{y}$, the shock front has to advance towards the inflowing gas, in order to decelerate the new inflowing gas. For the velocities relative to the shock, we have $u_{\mathrm{IN}}=v+\dot{y}$ and $u_{\mathrm{OUT}}=\dot{y}-\dot{z}$, so the isothermal shock condition gives

$\frac{v+\dot{y}}{a}=\frac{a}{\dot{y}-\dot{z}}$

$\dot{y}=\frac{\left[(v-\dot{z})^{2}+4 a^{2}\right]^{1 / 2}-(v-\dot{z})}{2}$.

The external pressure acting on the spheroid in the $z$-direction is therefore

$$
\begin{aligned}
P_{\mathrm{EXT}, z} & =\rho a^{2} \frac{v+\dot{y}}{\dot{y}-\dot{z}} \\
& =\rho a^{2} \frac{\left[(v-\dot{z})^{2}+4 a^{2}\right]^{1 / 2}+(v+\dot{z})}{\left[(v-\dot{z})^{2}+4 a^{2}\right]^{1 / 2}-(v+\dot{z})} .
\end{aligned}
$$

The external pressure acting on the spheroid in the $r$-direction is the same as in the unperturbed layer,

$$
P_{\mathrm{EXT}, r}=\rho a^{2} \frac{\left[v^{2}+4 a^{2}\right]^{1 / 2}+v}{\left[v^{2}+4 a^{2}\right]^{1 / 2}-v} .
$$

The rate of increase of the mass in the fragment, due to accretion from the continuing inflow, is

$$
\begin{aligned}
\dot{m} & =2 \pi r^{2} \rho(v+\dot{y}) \\
& =\pi r^{2} \rho\left\{\left[(v-\dot{z})^{2}+4 a^{2}\right]^{1 / 2}+(v+\dot{z})\right\},
\end{aligned}
$$

and we must add a term to equation (30) to represent the inertial drag of this accreted material, i.e.

$$
\begin{aligned}
\ddot{r} \simeq-\frac{3 G m}{2}\left\{\frac{r \cos ^{-1}(z / r)}{\left(r^{2}-z^{2}\right)^{3 / 2}}-\frac{(z / r)}{\left(r^{2}-z^{2}\right)}\right\} \\
-\frac{20 \pi P_{\mathrm{EXT}} r z}{3 m}+\frac{5 a^{2}}{r}-\frac{\dot{m} \dot{r}}{m} .
\end{aligned}
$$

The $P \mathrm{~d} V$ heating rate for the material already in the fragment is

$$
\mathcal{H}=-P \frac{\mathrm{d} V}{\mathrm{~d} t}=-m a^{2}\left[\frac{2 \dot{r}}{r}+\frac{\dot{z}}{z}\right]
$$

The heating rate due to dissipation of the $z$-kinetic energy of the matter accreting onto the fragment from the inflow, less the $r$-kinetic energy used to accelerate the accreted matter laterally, is

$\mathcal{D}=\frac{\dot{m}(v+\dot{z})^{2}}{2}-\frac{\dot{m} \dot{r}^{2}}{5}$

The maximum (i.e. blackbody) cooling rate from the two sides of the fragment is

$C(T)=2 \pi r^{2} \sigma_{\mathrm{SB}} T^{4}=\frac{2^{2} \pi^{6} \bar{m}^{4} a^{8} r^{2}}{15 c^{2} h^{3}}$,

where $h$ is Planck's constant and $\bar{m}$ is the mean gas-particle mass, as before.

\subsection{Survival criterion}

We can now follow the evolution of a collapsing fragment. The opacity limit for the proto-fragment is reached as soon as $\mathcal{H}+$ $\mathcal{D} \gtrsim \mathcal{C}(T)$. In reality, the radiative cooling is more complicated than Eq. (40). The gas flowing into the accretion shocks which define the top and bottom of the fragment (see Fig. 1) is initially heated to high temperature $\left(T_{\text {SноскеD }} \sim 3 \bar{m} v^{2} / 16 k_{\mathrm{B}}\right)$ in the shock front, and then rapidly cools behind the shock front. Provided we impose the condition $\rho v^{3} / 2 \ll \sigma_{\mathrm{SB}} T_{\text {SHOCKED }}^{4}$, or

$\rho \ll \rho_{\text {CRIT }} \equiv \frac{3^{3} \pi^{5} \bar{m}^{4} v^{5}}{2^{14} 5 c^{2} h^{3}} \simeq 10^{-12} \mathrm{~g} \mathrm{~cm}^{-3}\left[\frac{v}{\mathrm{~km} \mathrm{~s}^{-1}}\right]^{5}$,

then the immediate post-shock cooling (IPSC) radiation is either optically thin continuum radiation (e.g. from dust), or optically thick cooling in a few specific molecular lines which occupy a small total bandwidth. In either case, half the IPCS radiation is radiated away from the fragment, and half towards it. In the most extreme case, all of this latter half is absorbed by the fragment, and then has to be re-radiated at temperature $T$, along with the internal energy delivered by $P \mathrm{~d} V$ compression. Thus, by requiring the fragment to radiate at temperature $T$ all the energy dissipated in the accretion shock, we are making a rather conservative assumption.

To ensure that a proto-fragment forms a distinct condensation, i.e. that it continues to undergo contraction after the opacity limit has been reached, we set the following two survival conditions at the opacity limit:

1. the proto-fragment must be contracting in both the radial and vertical dimensions, i.e.

$$
\begin{aligned}
& \dot{r}_{\text {LIMIT }}<0, \\
& \dot{z}_{\text {LIMIT }}<0 ;
\end{aligned}
$$

2. the final extent of the proto-fragment, in both dimensions, must be less than or equal to half its initial radial size, i.e.

$$
\begin{aligned}
& r_{\text {LIMIT }}<0.5 r_{\text {INIT }}, \\
& z_{\text {LIMIT }}<0.5 r_{\text {INIT }} .
\end{aligned}
$$

For given $\rho, v$ and $a$ (i.e. for a given colliding flow), we must pick $t_{\mathrm{INIT}}$ and $r_{\mathrm{INIT}}$, where $t_{\mathrm{INIT}}$ is the time at which the fragment under consideration starts to condense out, and $r_{\mathrm{INTT}}$ is its initial radius. Thus the initial conditions for a trial fragment are

$$
\begin{aligned}
t & =t_{\mathrm{INIT}}, \\
r\left(t_{\mathrm{INIT}}\right) & =r_{\mathrm{INIT}}, \\
\dot{r}\left(t_{\mathrm{INIT}}\right) & =0, \\
z\left(t_{\mathrm{INIT}}\right) & =Z\left(t_{\mathrm{INIT}}\right), \\
\dot{z}\left(t_{\mathrm{INIT}}\right) & =\dot{Z}\left(t_{\mathrm{INIT}}\right) .
\end{aligned}
$$

We can then use Eqs. (37), (31), (34), (35) and (36) to follow the development of the fragment. Then we can use Eqs. (38)(40) to evaluate $\mathcal{H}, \mathcal{D}, \mathcal{C}$, and check whether the fragment has reached the opacity limit $(\mathcal{H}+\mathcal{D} \gtrsim \mathcal{C})$. Finally, once the limit is reached, we can check the survival conditions, Eqs. (42), (43), (44) and (45). 
In order to reduce the parameter space we fix $a=$ $0.2 \mathrm{~km} \mathrm{~s}^{-1}$, correponding to molecular gas at $10 \mathrm{~K}$. The equations are integrated using a fourth-order Runge-Kutta scheme.

In reality the gas in the two colliding streams will not have uniform density, and so $\rho$ should be interpreted as the mean pre-shock density. The presence of inhomogeneities in the preshock gas will promote fragmentation of the resulting layer, by creating the seed perturbations from which condensations subsequently grow. It will also tend to yield condensations with finite angular momentum. However, the lowest-mass condensations will be those with low angular momentum, and so we ignore angular momentum in this exploratory analysis.

\section{Results and discussion}

\subsection{General considerations}

A condensing fragment posseses two orthogonal dimensions $r$ and $z$. Moreover, (a) $r$ and $z$ in general have different initial values $\left(r\left(t_{\mathrm{INIT}}\right)\right.$ and $\left.z\left(t_{\mathrm{INIT}}\right)\right)$; (b) their excursions are driven by different external pressures (see Eqs. (34) and (35)); (c) the coupling between them (which occurs through both internal pressure and self-gravity) is non-linear. Therefore a fragment can evolve in quite complicated ways. In particular, small fragments which are not initially very unstable tend to oscillate until they accrete sufficient mass from the continuing inflow to become unstable against monotonic condensation. The oscillations in $r$ and $z$ normally have unrelated periods, and so the model has to be able to treat both oblate and prolate spheroids. However, once a fragment starts to condense out it is normally oblate, due to the extra ram pressure exerted in the $z$ dimension by the inflow.

\subsection{Oscillations}

If we fix $\rho$ and $v$, and vary $t_{\text {INIT }}$ and $r_{\text {INTT }}$, we find that the lowestmass condensation, $m_{\mathrm{MIN}}(\rho, v)$, forms when $t_{\mathrm{INIT}} \simeq t_{\mathrm{FRAG}}$ and $r_{\mathrm{INIT}} \simeq 0.5 r_{\mathrm{FRAG}}$. Fragments with $r_{\mathrm{INIT}} \lesssim 0.5 r_{\mathrm{FRAG}}$ tend to undergo radial oscillations before accumulating sufficient mass to condense out. Fragments with $r_{\mathrm{INT}}>0.5 r_{\mathrm{FRAG}}$ tend to condense out monotonically. The lowest-mass fragments are those which start with just enough mass to condense out monotonically, i.e. $r_{\mathrm{INIT}} \simeq 0.5 r_{\mathrm{FRAG}}$. Since $0.5 r_{\mathrm{FRAG}} \simeq R_{\mathrm{JEANS} 2}$, this finding agrees with the predictions of the linear stability analysis in Sect. 3.3.

\subsection{The optimum combination of $\rho$ and $v$ for producing low-mass condensations}

Suppose now that only $\rho$ is fixed, and $v$ is varied. For each $v$ we can determine the lowest-mass condensation $m_{\text {MIN }}(\rho, v)$, by varying $t_{\mathrm{INIT}}$ and $r_{\mathrm{INIT}}$ as described above. What we find is that as $v$ is increased at fixed $\rho, m_{\text {MIN }}(\rho, v)$ at first decreases, reaches a minimum, and then increases. Thus for any value of $\rho$ there is an optimum value of $v$ for spawning low-mass condensations. Figure 2 shows the $(\rho, v)$ plane and marks discrete points for which $m_{\text {MIN }}(\rho, v)$ is less than $0.005 M_{\odot} \equiv 5 M_{\text {JUPTER }}$. We see that there is a significant region of $(\rho, v)$ space in which condensations with mass below $0.005 M_{\odot}$ can form.

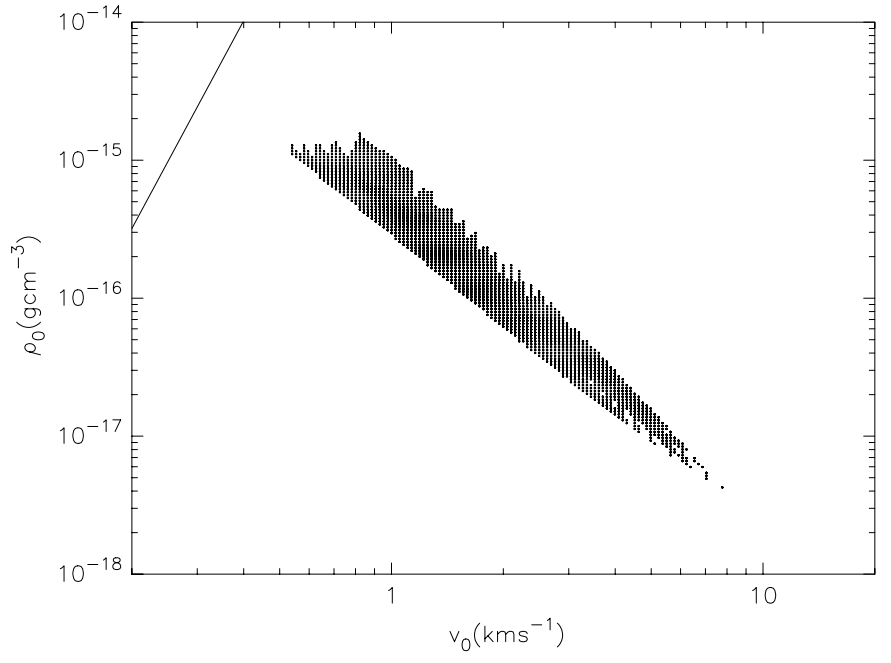

Fig. 2. A $\log / \log$ plot of the $(\rho, v)$ plane. The dots mark combinations of pre-shock density, $\rho$, and collision speed, $v$, for which (assuming a post-shock effective sound speed $a=0.2 \mathrm{~km} \mathrm{~s}^{-1}$, corresponding to molecular gas at $10 \mathrm{~K}$ ) the fastest-growing fragment mass is less than $0.005 M_{\odot}$. The irregularities in the boundary of this region have to do with the tendency of fragments with mass near the fastest growing one to undergo pulsations - see Sects. 4.2 and 4.3. The solid line is the locus (Eq. (41)) below which $\rho$ must fall if the immediate post-shock cooling radiation is to be negligible (as we assume).

This region has an irregular boundary because protofragments can undergo out-of-phase pulsations in the $r$ - and $z$-dimensions. If a proto-fragment undergoes a pulsation in the $r$-dimension and expands for a while $(\dot{r}>0)$, it experiences a prolonged period of growth due to accretion onto its relatively large cross-section $\left(\pi r^{2}\right)$, and hence its mass increases rapidly. Since the onset, amplitude and phase of such pulsations is critically dependent on initial conditions, small changes in $\rho$ and/or $v$ can result in quite large changes in $m_{\text {MIN }}$.

The full line on Fig. 2 shows the locus $\rho=\rho_{\text {CRIT }}=$ $10^{-12} \mathrm{~g} \mathrm{~cm}^{-3}\left[v / \mathrm{km} \mathrm{s}^{-1}\right]^{5}$. Hence the condition for the IPSC radiation to be neglected (Eq. (41)) requires $\rho$ to be well below this line. Evidently this condition is always easily fulfilled.

\subsection{The minimum mass}

The lowest-mass condensation of all is formed when $\rho \simeq 9.6 \times$ $10^{-16} \mathrm{~g} \mathrm{~cm}^{-3}, v \simeq 5.1 a \simeq 1.02 \mathrm{~km} \mathrm{~s}^{-1}, t_{\mathrm{INTT}}=t_{\mathrm{FRAG}} \simeq 1000 \mathrm{yr}$, and $r_{\text {INIT }}=0.62 r_{\text {FRAG }} \simeq 25 \mathrm{AU}$; the initial half-height of the fragment is $z\left(t_{\mathrm{INIT}}\right) \simeq 8 \mathrm{AU}$, and the initial mass of the fragment is $m\left(t_{\text {IIIT }}\right) \simeq 1.5 M_{\text {JUPTTER }}$. By the time the fragment has reached the opacity limit, its radius is $\sim 12 \mathrm{AU}$, and it is roughly spherical, but slightly oblate. $1600 \mathrm{yr}$ have passed since its inception, and its mass has increased by $1.1 M_{\text {JUPTTR }}$ to $2.6 M_{\text {JUPTTRR }}$. Thus $M_{\text {MIN2 }} \simeq 2.6 M_{\text {JUPTTRR }}$.

Figure 3 shows the development of this fragment. For the first $\sim 1200 \mathrm{yr}$, the fragment contracts in the radial dimension, but expands in the vertical dimension, due to accretion of extra material from the inflow into the layer. Around 1200 years after its inception the fragment starts to contract in the vertical dimension. At the same time there is a very small bounce in the radial dimension, but this is short-lived, and the 

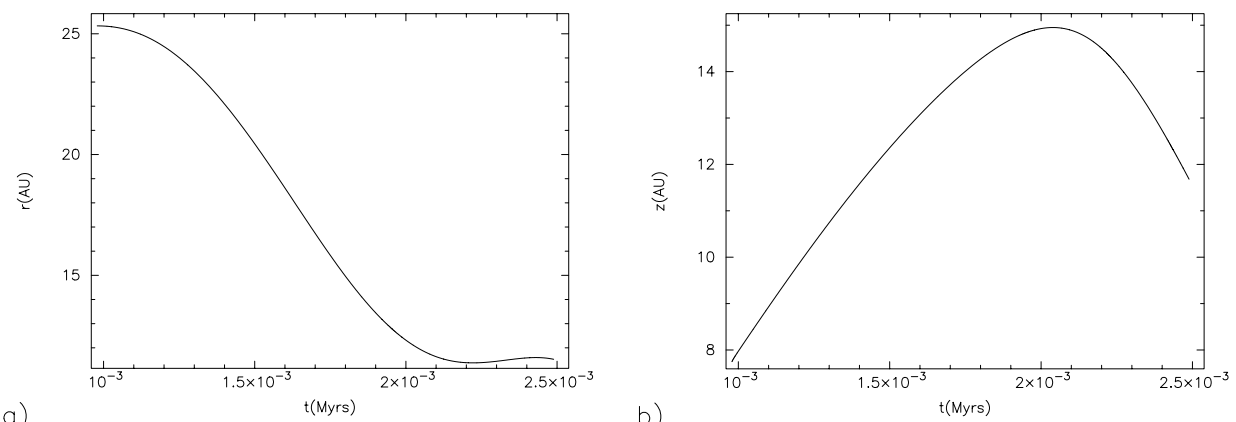

b)
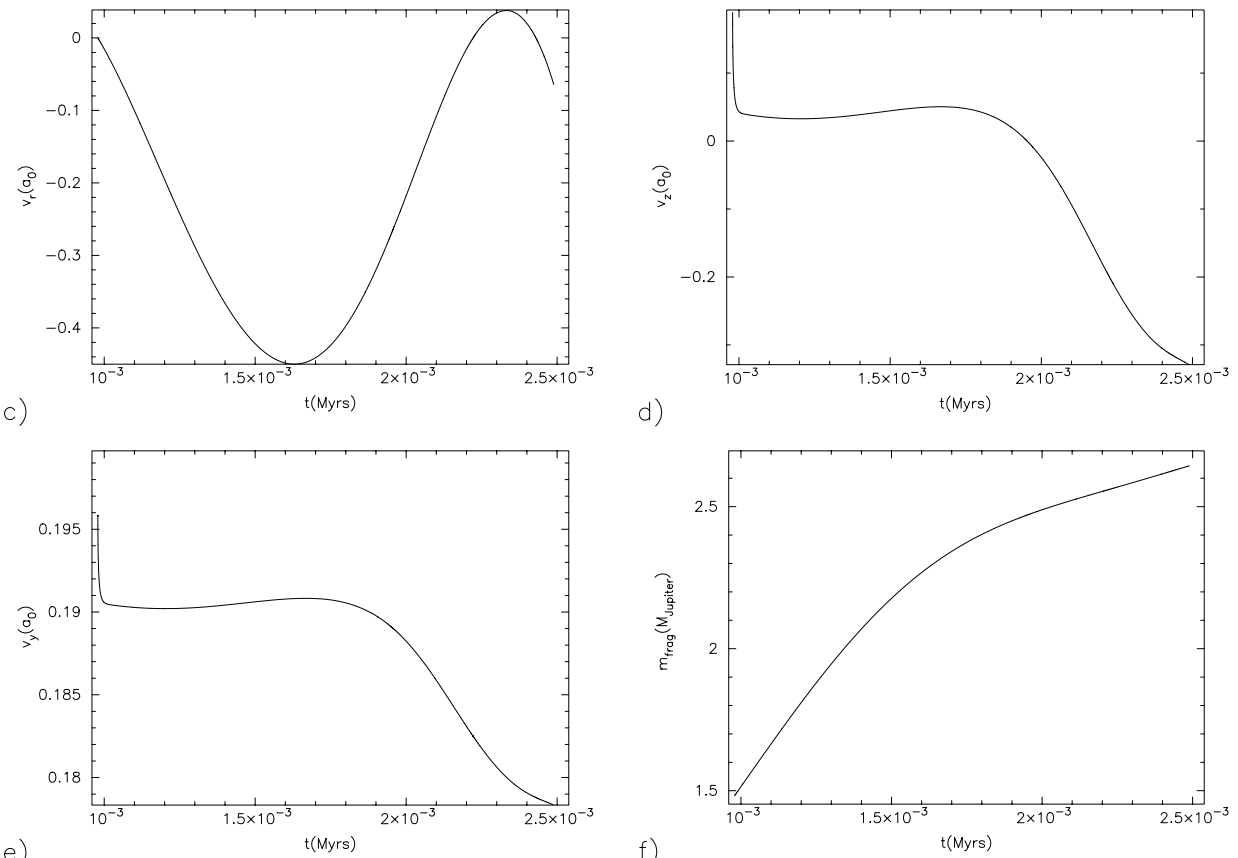

e)

f)
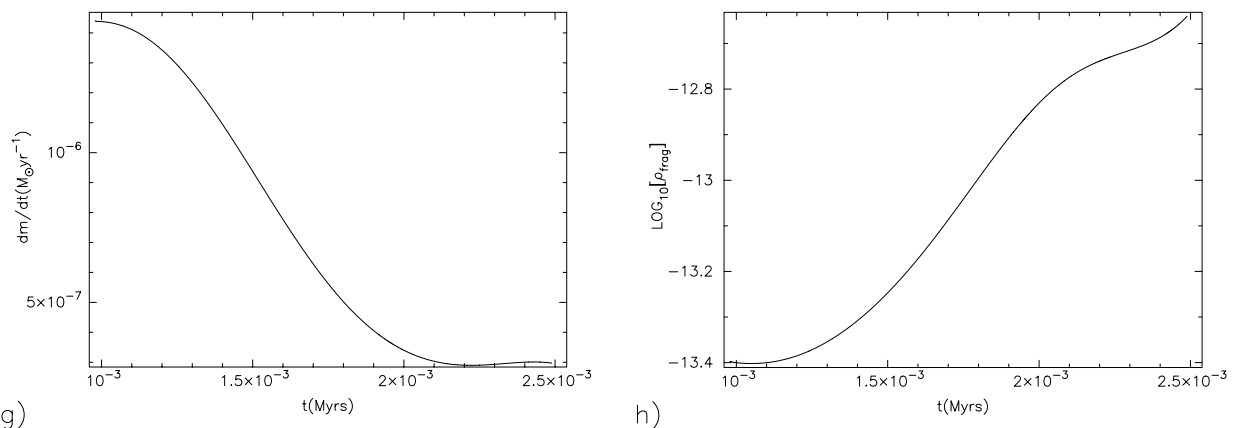

g)

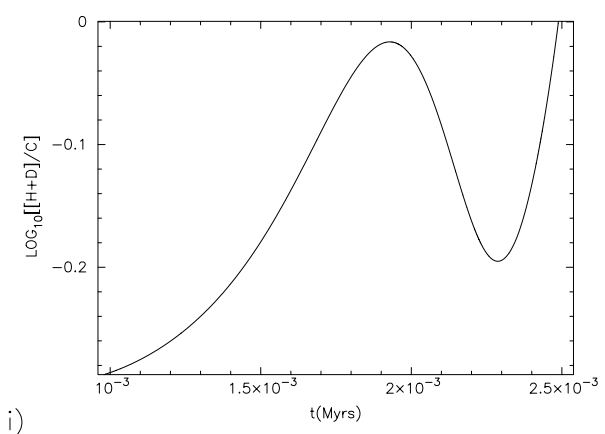

Fig. 3. Evolution of the minimum-mass fragment: a) radius, $r(t)$ in AU; b) height, $z(t)$ in AU; c) radial velocity, $\dot{r}(t)$ in units of $a=0.2 \mathrm{~km} \mathrm{~s}^{-1}$; d) vertical velocity of material already in the fragment, $\dot{z}(t)$ in units of $a$; e) vertical velocity of shock front bounding the fragment, $\dot{y}(t)$ in units of $a$; f) mass of fragment, $m(t)$ in $\left.M_{\text {JUPTER }} ; \mathbf{g}\right)$ accretion rate, $\mathrm{d} m / \mathrm{d} t$ in $\left.M_{\odot} \mathrm{yr}^{-1} ; \mathbf{h}\right)$ logarithmic fragment $\operatorname{density,} \ell \operatorname{og}\left(\rho_{\mathrm{FRAG}}\right)$ in $\mathrm{g} \mathrm{cm}^{-3}$; i) $\log$ arithmic ratio of heating to cooling, $\log ([\mathcal{H}+\mathcal{D}] / C)$. 
fragment quickly resumes its radial contraction. Soon after this the opacity limit is reached, and we must presume that the fragment then switches to Kelvin-Helmholtz contraction. At this stage the fragment is roughly spherical. It is still accreting, at a rate $\sim 2 \times 10^{-4} M_{\text {JUPTTER }} \mathrm{yr}^{-1}$, and it has a dynamical timescale of $\sim 1000 \mathrm{yr}$, so we might expect its mass to increase by a further few tenths of a Jupiter mass during the subsequent condensation.

\section{Conclusions}

In 3-dimensional hierarchical fragmentation, the minimum mass is estimated to be $M_{\text {MIN3 }} \sim 7$ to $10 M_{\text {JUPITER }}$ (e.g. $7 M_{\text {JUPITER }}$, Low \& Lynden-Bell 1976, $10 M_{\text {JUPITER }}$, Silk 1977; $10 M_{\text {JUPITER }}$, Boss 1988). Moreover, these estimates probably need to be revised upwards, to take account of ongoing accretion and merging during concensation (see Sect. 2).

However, surveys of young clusters are beginning to find objects with masses estimated to be as low as $3 M_{\text {JUPITER }}$ (e.g. Zapatero Osorio et al. 2002).

We find that, in the 2-dimensional fragmentation of a shock compressed layer, the minimum mass is significantly smaller than $10 M_{\text {JUPITER }}$. For certain rather specific shock parameters, fragments with mass $<3 M_{\text {JUPTTR }}$ can condense out - specifically $M_{\text {MIN2 }} \simeq 2.6 M_{\text {JUPTTER }}$ - and for a wide range of shock parameters fragments with $\lesssim 5 M_{\text {JUPTTR }}$ can condense out. Since (i) layer fragmentation is probably a more realistic model for fragmentation in highly turbulent, transient star-forming clouds; (ii) layer fragmentation is more likely to be permanent than 3-dimensional fragmentation (because the fragments in a layer condense out faster than the layer as a whole); and (iii) our analysis takes proper account of ongoing accretion during fragmentation, we conclude that free-floating planetary-mass objects can form by the fragmentation of a shock-compressed layer, in the same way as stars and brown dwarves.

Acknowledgements. D.F.A.B. thanks PPARC for the support of a postgraduate studentship. A.P.W. thanks the European Commission for support under contract HPRN-CT-2000-00155.

\section{References}

Bhattal, A. S., Francis, N., Watkins, S. J., \& Whitworth, A. P. 1998, MNRAS, 297, 435

Boffin, H. M. J., Watkins, S. J., Bhattal, A. S., Francis, N., \& Whitworth, A. P. 1998, MNRAS, 300, 1189

Boss, A. P. 1988, ApJ, 331, 370

Elmegreen, B. G. 2000, ApJ, 530, 277

Elmegreen, B. G. 2002, IAU Symp., 207, 390

Hartmann, L., Ballesteros-Paredes, J., \& Bergin, E. A. 2001, ApJ, 562, 852

Hester, J. J. 1997, in Star Formation Near and Far, ed. S. S. Holt, \& L. G. Mundy, American Institute of Physics, Conf. Proc., 393, 143 Hoyle, F. 1953, ApJ, 118, 513

Larson, R. B. 1981, MNRAS, 194, 809

Larson, R. B. 1985, MNRAS, 214, 379

Low, C., \& Lynden-Bell, D. 1976, MNRAS, 176, 367

Mac Low, M.-M., \& Klessen, R. S. 2004, Rev. Mod. Phys., 76, 125

Padoan, P. \& Nordlund, Å. 2002, ApJ, 576, 870

Pringle, J. E., Allen, R. J., \& Lubow, S. H. 2001, MNRAS, 327, 663

Rees M. J. 1976, MNRAS, 176, 483

Silk, J. 1977, ApJ, 214, 152

Turner, J. A., Chapman, S. J., Bhattal, A. S., et al. 1995, MNRAS, 277,705

Vázquez-Semadeni, E., Ballesteros-Paredes, J., \& Klessen, R. 2003, ApJ, 585, L131

Watkins, S. J., Bhattal, A. S., Boffin, H. M. J., Francis, N., \& Whitworth, A. P. 1998a, MNRAS, 300, 1205

Watkins, S. J., Bhattal, A. S., Boffin, H. M. J., Francis, N., \& Whitworth, A. P. 1998b, MNRAS, 300, 1214

Whitworth, A. P., Bhattal, A. S., Chapman, S. J., Disney, M. J., \& Turner, J. A. 1994a, A\&A, 290, 421

Whitworth, A. P., Bhattal, A. S., Chapman, S. J., Disney, M. J., \& Turner, J. A. 1994b, MNRAS, 268, 291

Whitworth, A. P., Chapman, S. J., Bhattal, A. S., et al. 1995, MNRAS, 277, 727

Whitworth, A. P., \& Zinnecker, H. 2004, A\&A, 427, 299

Zapatero Osorio, M. R., Béjar, V. J. S., Martín, E. L., et al. 2002, ApJ, 578,536

Zeldovich, Ya. B. 1970, A\&A, 5, 84 